\title{
Performance Improvement of OFDM Systems by Reducing Papr using SLM-Grasshopper Optimization
}

\author{
Reddi Sridevi, T.Madhavi, Sreenivasulu Ummadisetty
}

\begin{abstract}
Orthogonal Frequency Division Multiplexing (OFDM) is an emerging technology which is been most commonly used for transmission of data in wireless based systems. The OFDM technique is capable of achieving more speed i.e it has higher data rate transmission which is reliable and efficient. Peak power is the major drawback while transmitting the signal which is termed as PAPR. The signals achieves high peak value while transmitting due to the presence of large subcarriers which are independent in nature. The amplitude of the signal need to be reduced. Many PAPR reductions schemes have been presented in past and the reduction is done to some extents. In this paper, a new optimisation technique i.e grasshopper optimization (GOA) is proposed. The optimization technique is combined with one of the efficient PAPR reduction technique i.e selective mapping technique. The combination of SLM-Grasshopper help in reducing the PAPR as low as possible and is also compared with other techniques like SLM-GA, SLM-FA and SLM-GWO. The experimental results are performed using matlab.
\end{abstract}

\section{Keywords: ODFM, PAPR, SLM, Grasshopper Algorithm}

\section{INTRODUCTION}

Orthogonal frequency division multiplexing (OFDM) is a system which have multiple subcarriers and modulation method which looks to be an captivating candidate for fourth generation $(4 \mathrm{G})$ wi-fi verbal exchange systems. The system have good level of spectral form which is efficient in nature, immune to the multipath delay, the interference present while transmitting the data is low. The system is immune to frequency selective fading and the efficiency level fo power is excessive records charge communication systems such as Digital Video Broadcasting (DVB) and based cellular worldwide makes use for microwave get entry to the wi-max systems. However OFDM device has a major level of problem that is increase in PAPR.

In OFDM device output is superposition of a couple of sub-carriers. Contingent upon some of the spot energy output may amplify appreciably and emerge as a ways higher than the power that is utilised of the system. High PAPR signals

need to be transmitted in some cases which should have a minimum requirement of electricity amplifier with high level scope of the power[1]. The degradation of performance of

Revised Manuscript Received on December 13, 2019.

* Correspondence Author

Reddi Sridevi,, Assistant Professor, Department of ECE, Dr.L.B College of Engineering, Visakhapatnam, India.Email:himasri_sss@yahoo.com

Dr.T.Madhavi, Professor, Dept. of EECE, GIT, GITAM (Deemed to Be University), Visakhapatnam, India. Email:madhavi.tatineni@gitam.edu

Sreenivasulu Ummadisetty, Assistant Professor, Dept. of EECE, GIT, GITAM (Deemed to Be University), Visakhapatnam, India. Email:sreenuaswarth@gmail.com efficient. As OFDM have many merits it is chosen as

OFDM is due to the quick distortions in the non linear form which adjustments the spectrum signals postions[2]. If no measure is taken to decrease the excessive PAPR, MIMO-OFDM systems ought to face serious restriction for practical functions [3][4].

Complementary cumulative distribution characteristic (CCDF) is used to describe PAPR. The researchers have been developed and proposed probabilistic method positive schemes. These include clipping, coding and sign scrambling techniques. Partial transmit sequence (PTS) and Selected Mapping (SLM) are the two popular schemes under the heading of signal scrambling techniques. Some of the methods that are used to reduce the PAPR are determined and summarized in [5], it is nevertheless indeed wanted to provide some of the stratagies that are used to reduce the PAPR has a comprehensive evaluation, such as electricity saving, and to evaluate some typical methods of PAPR discount via theoretical evaluation and simulation effects thoroughly.

Selective mapping method is one of the promising PAPR reduction technique without any distortions in the signals. A new approach of PAPR reduction method is applying genetic optimization to the SLM . GA is applied to SLM-OFDM system, by which the random populations are considered whose fitness values are evaluated for looking the most appropriate section rotation elements and decreasing computational complexity[6].

A new technique for solving core problem of OFDM by reducing the PAPR. The technique proposed is Firefly optimization which is applied along with selective mapping technique in which rotation of phase factor for search was compared with initial OFDM, conventional SLM-OFDM and also compared with genetic algorithm based SLM[7]

Further, Grey wolf optimization technique is combined with SLM to reduce the PAPR, the grey wolf optimization the PAPR reduce to some extent. More studies need to be emerged to reduce the PAPR. There is much better reduction in PAPR value using grey wolf by which the problem of transmitting signal is minimised[8].

In this paper, the performance and the traits of ODFM system is observed. A new PAPR reduction technique is implemented. The behaviour grasshopper is studied. Finally SLM is combined with grasshopper optimization algorithm to achieve good rate of decrease in PAPR.

\section{OFDM AND PAPR}

In OFDM system the signal which is transferred from source to destination is made of subcarriers which are modulated with the aid of constellations mapping. 
The modulation techniques like QAM and PSK are used for performing the function of mapping and are process is been completed. For an OFDM device with $\mathrm{N}$ sub-carriers, the high-speed binary serial enter circulate is denoted as $\left\{q_{\mathrm{i}}\right\}$. After conversion of signal from serial to parallel (S/P) and process of mapping, a new parallel sign sequence is obtained and given as,

$\left\{a_{0,}, a_{1}, a_{2, \ldots \ldots,} a_{i, \ldots \ldots \ldots,} a_{N-1}\right\}$ is obtained, $a_{i}$ is termed as sign value of discrete signal[6]. Each element of parallel signal sequence is supplied to $\mathrm{N}$ orthogonal sub-carriers is given as,

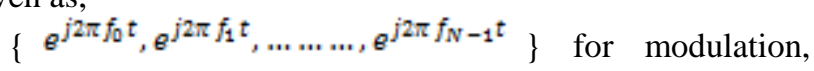
respectively. The signals which are modulated are added together to form an OFDM symbol. The system structure of OFDM is been simplified with the use of discrete Fourier transform. The transmitted OFDM signals can be written as

$$
x(t)=\frac{1}{\sqrt{N}} \sum_{k=0}^{N-1} X_{k} e^{j 2 \pi f_{k} t}, 0 \leq t \leq N T
$$

Probability Density Function (PDF) for a signals with large value of $\mathrm{N}$ is given by [5].

$$
\begin{aligned}
& P_{\gamma}\{x(t)\}=\frac{1}{\sqrt{2 \pi \sigma}} e^{-\frac{[x(t)]^{2}}{2 \sigma^{2}}} \\
& \text { where } \sigma \text { is the variance of } \mathrm{x}(\mathrm{t})
\end{aligned}
$$

\section{PAPR}

The signal is considered as $\mathrm{x}(\mathrm{t})$, for a given $\mathrm{x}(\mathrm{t})$ the PAPR is calculated and is defined as maximum power obtained during the transmitting of signal to its average range of power[3].

$$
\operatorname{PAPR}[x(t)]=\frac{P_{\text {PEAK }}}{P_{\text {AVERACE }}}=10^{\log _{10} \frac{\left.\max [\mid x(n)]^{2}\right]}{E\left[|X(n)|^{2}\right]}}
$$

where $P_{\text {PEAK }}$ - peak power at output,

$P_{\text {AVERAGE - average output power, the expected value can }}$ be termed as $\mathrm{E}[\cdot]$, By performing the IFFT operation on the signals which are modulated to obtain the signal and which is a need that to be transmitted signals in OFDM with symbols $X_{k}$ is given as $x_{n}[11] . x_{n}$ is expressed as:

$$
x_{\mathrm{n}}=\frac{1}{\sqrt{\mathrm{N}}} \sum_{K=0}^{\mathrm{N}-1} X_{k} W_{N}^{\mathrm{nk}}
$$

when compared with normal single carrier systems, the output achieved from an OFDM system has high fluctuations in a regular manner. Due to which the system requires an power amplifier, converters etc. If the condition is not satisfied an undesirable inference will be encountered and the signal moves into non linear region at the transmitter side, which is called as distortion due to inter modulation which have high radiations. In OFDM system reducing of PAPR is an incredible process.

\section{SELECTED MAPPING (SLM)}

The approach of SLM is shown in Figure 1, from a single OFDM system, the sequence is presented as D and the length of the sequence is denoted as N. Based on the number of sequences generated, the element rotation will be defined. Here, the consideration of sequence and rotation is equal. So the signal which is transmitted has lowest PAPR. The generation of new sequence is performed and is termed to be $\mathrm{M}$, which is called as length of SLM. The sequences which are obtained are the result of multiplying the incoming authentic sequence of OFDM 'A' with the aid of $\mathrm{M}$ exclusive rotation factors. The vector shapes are provided for the elements [9],
$B^{(\mathrm{i})}=b_{0}^{(\mathrm{i})}, b_{1}^{(\mathrm{i})}$ $b_{\mathbb{N}-1}^{(1)}$

where $i$ represents the indices of these factors with $\mathrm{i}=1$ to $\mathrm{M}$ and representation $\mathrm{B}$ is known as rotation factor in the form of vector. The original OFDM sequence $\mathrm{D}$ is multiplied with rotation factor and we get:

$X^{(\mathrm{i})}=a_{0} b_{0}^{(\mathrm{i})}, a_{1} b_{1}^{(\mathrm{d})}, \ldots \ldots \ldots \ldots \ldots a_{N-1} b_{N-1}^{(\mathrm{i})}$

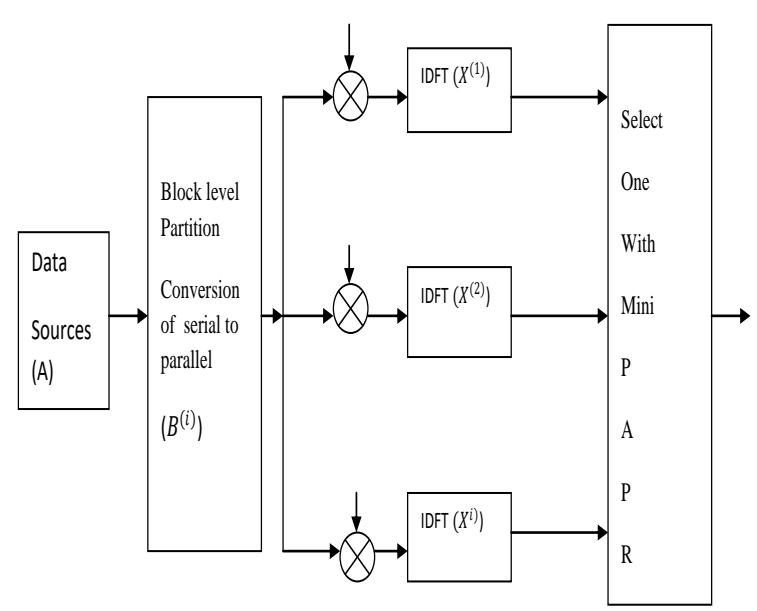

Fig.1. SLM block at the end of transmitter [7]

In this phases are viewed and they rotate for this reason based totally on the inputs generated. They multiply a complicated number with the rotating factor and find the one more number. The decision process will be endured until mini PAPR is achieved. The calculations are performed at every section [5]. Hence,

$b_{n}^{i}=e^{-j \theta_{n}^{i}}$ where $\theta_{n}^{(i)} \in[0,2 \pi]$

where $\theta$ is the phase rotation. These vectors are used to recover the signal that are used as facet records which are been transmitted. The amount of scrambling performed resembles the effectiveness and efficiency SLM. The scrambling is done with help of rotation factors on the unique sequence of OFDM and the length of SLM sequence. Here the length of the SLM is viewed as $U$ and the level of complexity is calculated. The overall performance of SLM is evaluated based on the quantity of iterations or sequences and by the way of increasing the sequence the system complexity may additionally expand but the most important hassle which is considered in transmitting give up i.e PAPR can be minimize to some extent. The complexity of imposing SLM is termed to be moderate. Some works have been preferred to limit the stage of complexity in SLM. The complexity of SLM can be termed as

$$
\text { Complexity }=U N \operatorname{Uog}_{2} N
$$

\section{SLM-GRASSHOPPER OPTIMIZATION}

It is one of the famous optimization technique proposed by mirjalili and inspired by swarm optimization model. The existence of grasshoppers life process is shown in Figure. 2 . Although grasshoppers are generally considered for my part in nature, they be a part of in one of the biggest swarm among all the insects [12]

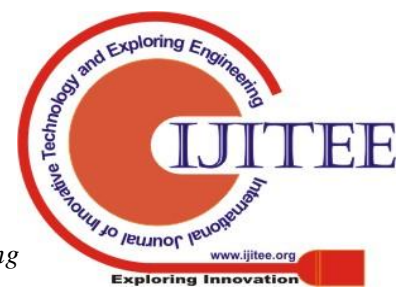


The grasshoppers has a unique feature in nymph and adulthood is its swarming behaviour [13]. As nymph is the starting stage of grasshoppers life cycle, even it is observed that Millions of them move and sour. In their path, they devour nearly all vegetation. Later, at the end of adult stage the grasshoppers develop a swarm in air. The way how grasshoppers migrate over massive routes covering long distances. One of the major trait of the swarm is its larval condition in which it has slow movement with small steps. The larger distance and the movement which is very much abrupt is the indispensable characteristic of the swarm in stage of adulthood. Food source in search of is every other vital attribute of the swarming of grasshoppers. The search process is performed using two types of tendencies which are separated logically and are termed as: exploration and exploitation. In case of exploration, the search sellers are motivated to go abruptly, in the course of exploitation local crossing is observed. The target seeking functions are carried out in two function by the mean of natural behaviour of grasshopper.

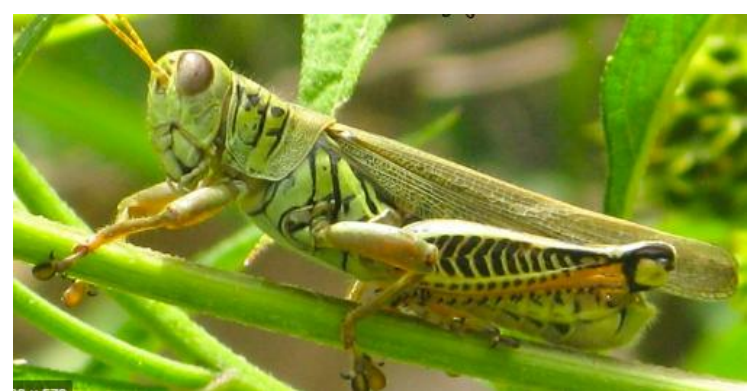

(a) original grasshopper

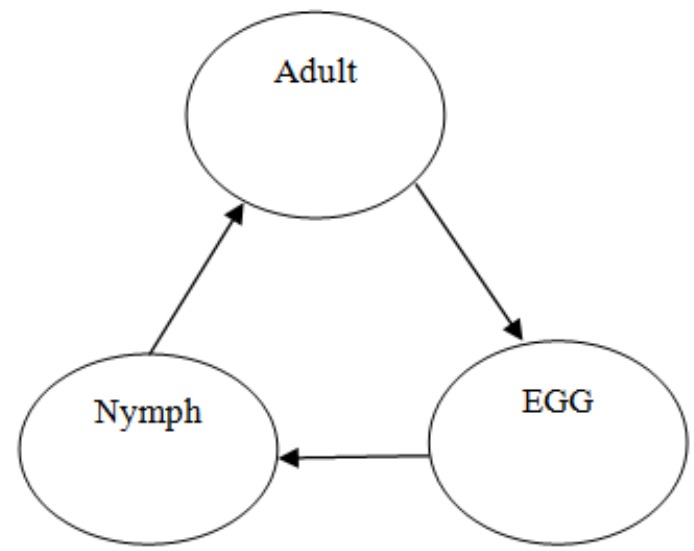

(b) grasshopper life process

Fig. 2. Grasshopper and process of its life

The model representation for simulation of the behaviour of grasshopper that are swarming are given as[14]

$X_{\mathrm{i}}=S_{\mathrm{i}}+G_{\mathrm{i}}+A_{\mathrm{i}}$

position of $i^{\text {th }}$ grasshopper is $X_{i}$, the interaction of grasshoppers socially is $S_{i}$, the $i^{\text {th }}$ grasshopper gravitational force is $G_{i}$, and the direction of wind which the grasshoppers moves is $A_{\mathrm{i}}$. The random behaviour of grasshoppers with various conditions is written as $X_{i}=r_{1} S_{i}+r_{2} G_{i}+r_{a} A_{i}$ where $r_{1}, r_{2}$ and $r_{a}$ are random numbers in $[0,1]$.

$s_{i}=\sum_{\substack{j=1 \\ j \neq 1}}^{\mathbb{N}} s\left(d_{i j}\right) \widehat{d_{i j}}$

The distance between $i^{\text {th }}$ grasshopper and $i^{\text {th }}$ grasshopper is given as $d_{i j}$ and is calculated as, $d_{i j}=\left|x_{j}-x_{i}\right|$ and $\widehat{d_{i j}}=\frac{x_{j}-x_{i}}{d_{i j}}, \mathrm{~s}$ is termed as social force which defines the strength of grasshopper socially.

A conceptual mannequin of the interactions between grasshoppers and the relief area the use of the characteristic s. It may additionally be stated that, in simplified form, this social interplay used to be the motivating pressure in some until now locust swarming models [15]. The house of grasshoppers is divided into three zones by function $\mathrm{S}$. The zone are named as comfort zone, repulsion zone and enhancement zone. if the distance between the two grasshoppers is above 10 then the value is set to zero. When the value is set to zero the grasshopper is not able to follow the study forces that are described above. The distance between the grasshopper are calculate for every update of the positions.

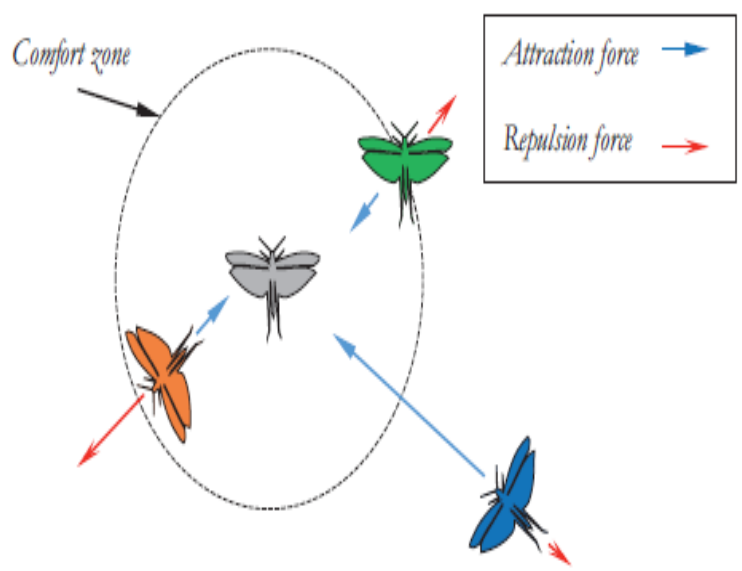

Fig.3. Zones and behaviour of grasshoppers

The component $\mathrm{G}$ is given as,

$G_{i}=-g \hat{e_{g}}$

here $\mathrm{g}$ is termed as constant of gravitation and the unit vector is $\widehat{e_{g}}$ which acts moving towards the centroid position of the earth .

The component $\mathrm{A}$ is given as,

$A_{\mathrm{i}}=u \widehat{e_{W}}$

The expanded equation by including all the components is given by

$X_{i}=\sum_{\substack{j=1 \\ J \neq 1}}^{W} s\left(\left|x_{j}-x_{i}\right|\right) \frac{x_{j j}-x_{i}}{d_{i j}}-g \widehat{e_{g}}+u \widehat{e_{i W}}$

The grasshoppers are very quick in reaching the comfort zone , so that the opimization problem cannot be solved accurately. Inorder to solve the optimizations problem the modified version of the equation 13 is given as

$X_{i}^{d}=c\left(\sum_{\substack{j=1 \\ j \neq 1}}^{N} c \frac{w b_{d}-i b_{d}}{2} s\left(\left|x_{j}^{d}-x_{i}^{d}\right|\right) \frac{x_{j}-x_{i}}{d_{i j}}\right)+\widehat{T_{d}}$ 


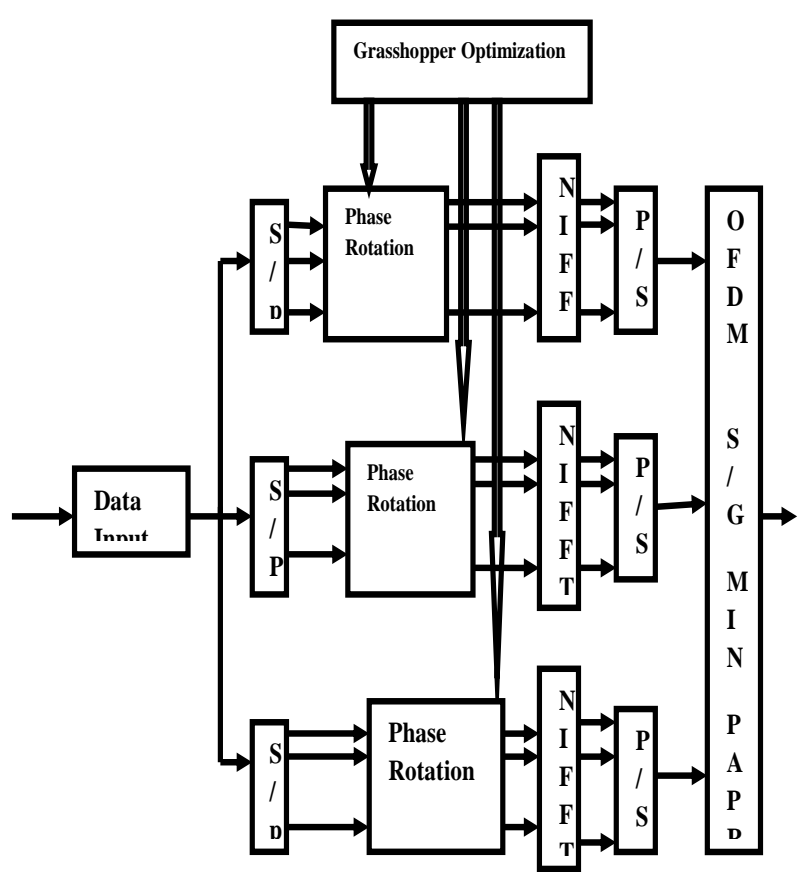

Fig.4. Proposed SLM-Grasshopper Model

The role of grasshopper relies on the sting position, target position and eventually the position of all grasshoppers that is shown in eq (14). Note that the primary side of this equation considers the locality of the up to date grasshopper with appreciate to different grasshoppers. The grasshoppers quality is taken into account to stipulate the search sellers spherical region. In GOA, the search is performed position vectors of every search agent. supported the present position, the search agents operate is updated and more updates to next position supported international best, and therefore the role of all totally different search agents. The state involves all the search dealers in shaping the position of the search agent that is extremely distinctive feature to provide optimized results.

\section{A. Algorithm}

The grasshopper optimization based SLM algorithm is given as:

Step1: Initialise input data $l_{x}=I_{1} I_{2} I_{3 \ldots \ldots \ldots \ldots} \ldots I_{n}$ Step2: Serial bit of data converted to parallel bits

Step3. Rotating the phase of bits and applying Grasshopper optimization for the bits

Step 4. Initialization of the search agents nymph and adult grasshopper

Step5: Calculate the fitness function

Step6: Find the best grasshopper among nymph and adult

Step7: Update the position of nymph w $\mathrm{r} t$ Social interaction, gravitation force and wind advection Step8: Calculate the distances between nymph and adult grasshopper

Step9: update the position and calculate the fitness value of nymph and adult

Step10. Find the best value and form a unique phases

Step11. Obtain the Min PAPR signal

\section{RESULTS AND DISCUSSION}

The results obtained are shown in below Figures. From Figure 4, more reduction in PAPR is observed for SLM-Grasshopper method than the techniques which are used o compared. Hence the OFDM is a bit free form power factor issue by the proposed method and also better compared to other techniques like SLM, GA-SLM, FA-SLM, GWO-SLM. The results shows the variation in PAPR with respect to the CCDF for different phase sequences like 4, 8, 16 and 32. Here the phase sequences is denoted with $M$. As the $M$ value increases the PAPR get reduced and remains stable after reaching certain point. From the experimental results it is shown that the PAPR is no further reduced for $\mathrm{M}$ value above 32. There is no use in increasing the phase value as the system level of complexity increases with increase in number of phases. By considering $\mathrm{M}=4$ to $\mathrm{M}=32,0.5 \mathrm{~dB}$ decrease is observed in each case.

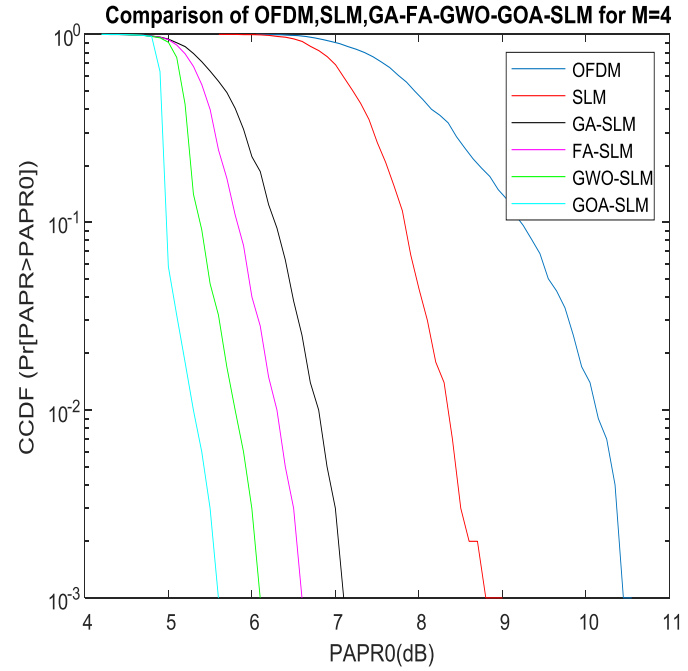

Fig.5. PAPR vs CCDF for $M=4$

For $\mathrm{M}=8$,

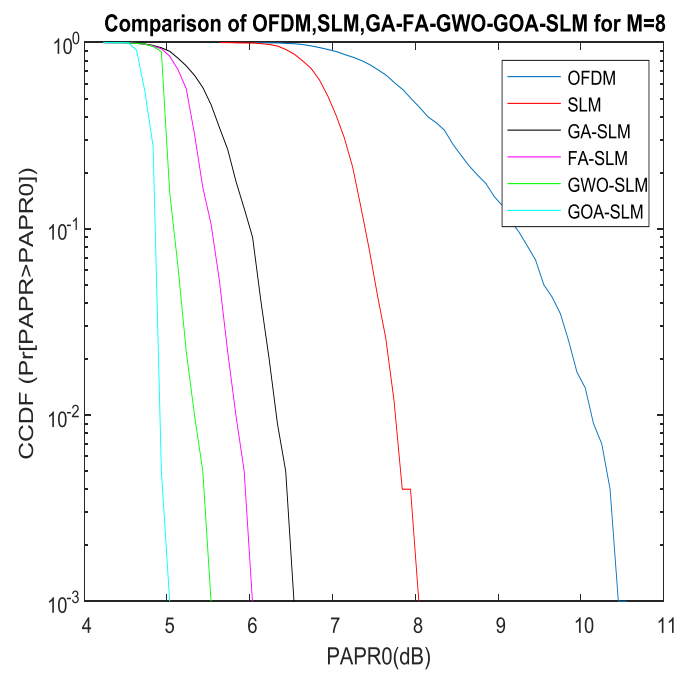

Fig.6. PAPR vs CCDF for $\mathrm{M}=8$

As shown in Figure 7, for 16 phases by estimating the global value the best optimal value for PAPR found by GOA is : 4.6371 


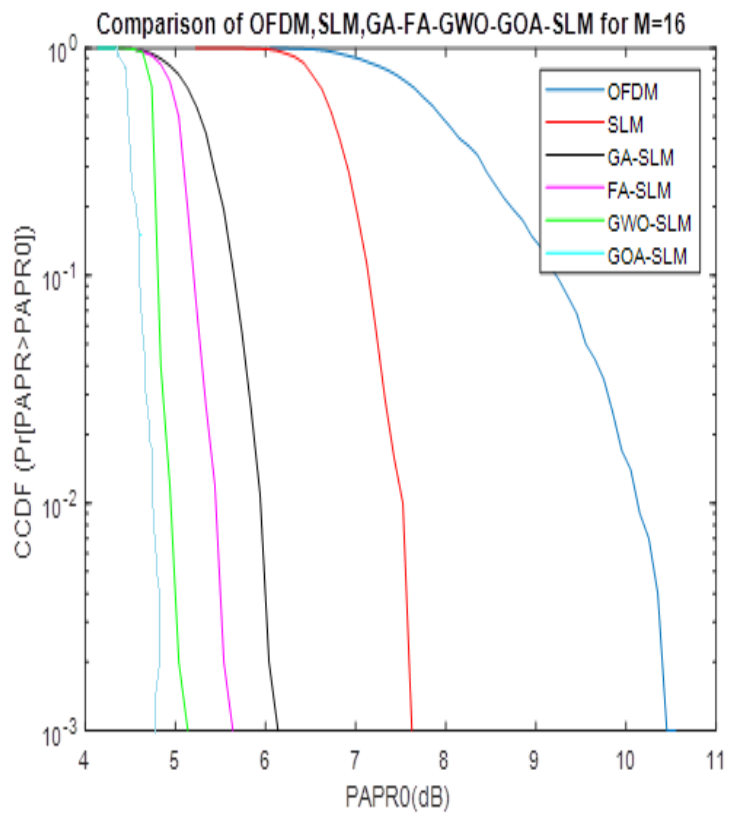

Fig.7. PAPR vs CCDF for $\mathrm{M}=16$

As the number of number of phases increases the PAPR reduces and some breakeven point the PAPR value remains constant. The PAPR optimal value obtained for $\mathrm{M}=32$ while performing with GOA is 4.4 which is shown in Figure 8 .

\section{CONCLUSION}

In terms of spectrum efficiency and the performance of channel OFDM is a technique which is greater pinching in wi-fi communications. The only primary disadvantage need to think about is its PAPR. It achieves greater PAPR when the number of subcarriers increases. One of the useful scrambling approach i.e SLM and most efficient optimization technique i.e grasshopper algorithm together mixed to decrease PAPR to increase the entire performance of OFDM communication system. The decrease in PAPR is proven with amplify in phase sequence $\mathrm{M}$. The overall performance of proposed approach is in contrast with many present strategies and proved to be best. The experimental outcomes shows that SLM-Grasshopper algorithm outperforms and the sizable adjustments in PAPR is observed.

\section{REFERENCES}

1. Y.Wu and W. Y. Zou, "Orthogonal frequency division multiplexing: A multi-carrier modulation scheme," IEEE Trans. Consumer Electronics, vol. 41, no. 3, pp. 392-399, Aug. 1995.

2. U1niversity of Alberta, "Home page - High capacity digital communications laboratory,” $2007 . \quad$ Available: http://www.ece.ualberta.ca/ HCDC/mimohistory.html.

3. Shinsuke Hara, Ramjee, "Principle and history of MCM/OFDM," in Multicarrier techniques for 4G mobile communication, Artech House.

4. E. Telatar, "Capacity of multi-antenna Gaussian channels," European Transactions on Telecommunications, vol. 10, no 3, Dec 1999.

5. Foschini G J, Gans M J, "On limits of wireless communication in a fading environment when using multiple antennas," Wireless Personal Communication, vol. 6.

6. Tarik Hadj Ali and A. Hamza, " A new SLM technique based on Genetic Algorithms for PAPR reduction in OFDM systems", IEEE DOI 10.1109/CICN.2016.113DOI

7. R Sridevi and Dr. T Madhavi, "SLM Technique based on Firefly Algorithm for Reduction of PAPR in OFDM Systems ", Jour of Adv Research in Dynamical \& Control Systems, Vol. 11, 06-Special Issue, 2019.

8. R Sridevi and Dr. T Madhavi, " Reduction of PAPR in OFDM Signals Using Grey Wolf Optimization combined with SLM ", DSMLA 2019: Proceedings of the 1st International Conference on Data Science, Machine Learning and Applications.

9. Suverna Sengar, Partha Pratim Bhattacharya," Performance Improvement In OFDM system By PAPR Reduction," Signal \& Image Processing : An International Journal (SIPIJ) Vol.3, No.2, April 2012 Department of Electronics and Communication Engineering, Faculty of Engineering and Technology, Mody Institute of Technology \& Science (Deemed University),Lakshmangarh, Dist Sikar, Rajasthan, Pin - 332311, INDIA.

10. Chin-Liang Wang, Yuan Ouyang, "Low-complexity selected mapping schemes for peakto- average power ratio reduction in OFDM systems", IEEE Trans. on Signal Processing, vol. 53, no. 12, pp. 4652 - 4660, 2005.

11. Peled A, Ruiz A, "Frequency domain data transmission using reduced computational complexity algorithms," Acoustics, Speech, and Signal Processing, IEEE International Conference.

12. Simpson SJ , McCaffery A, HAeGELE BF . A behavioural analysis of phase change in the desert locust. Biol Rev 1999;74:461-80

13. Rogers SM , Matheson T, Despland E, Dodgson T, Burrows M , Simpson SJ . Mechanosensory-induced behavioural gregarization in the desert locust Schis- tocerca gregaria. J Exp Biol 2003;206:3991-4002 .

14. Topaz CM, BernoffAJ, Logan S, Toolson W . A model for rolling swarms of lo- custs. Eur Phys J Special Top 2008;157:93-109 .

15. Lewis A . LoCost: a spatial social network algorithm for multi-objective optimisation. In :Evolutionary computation, 2009. CEC'09. IEEE congress on; 2009. p. 2866-70.

\section{AUTHORS PROFILE}

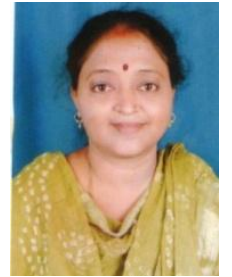

systems

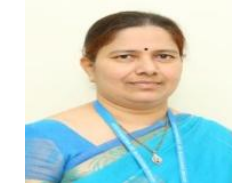

Dr. T. Madhavi is currently working as Professor in the Department of EECE, GITAM, Visakhapatnam, India. She received her M.Tech in Radar and Microwave Engineering in 2004 and Ph.D in 2013 from Andhra University, India. She has over 25 years of experience in Industry and Teaching. She has published several papers in Reputed Journals like IET, Springer, etc. Her main research interest includes modeling and performance analysis of wireless communications systems, Cellular/mobile communications and Wireless Sensor Networks

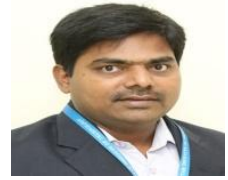

SREENIVASULU.UMMADISETTY received the B.Tech degree in Electronics Instrumentation and Control Engineering from S.V .University, Tirupati,A.P., India in 2007, M.Tech degree from NIT Rourkela, Orissa, India in 2010. He is currently working as Assistant Professor, Dept. of EECE, GIT,GITAM University, Visakhapatnam, AP, India. His Research interests include Bio medical Signal Processing, Radar Signal Processing, soft computing applications. 